\title{
ON HOW KELSENIAN JURISPRUDENCE AND INTUITIONISTIC LOGIC HELP TO AVOID CONTRARY-TO-DUTY PARADOXES IN LEGAL ONTOLOGIES
}

\author{
Edward H. Haeusler ${ }^{1} \&$ Alexandre Rademaker ${ }^{2}$
}

\begin{abstract}
In this article we show how Hans Kelsen jurisprudence and Intuitionistic logic are used to avoid the well-known contrary-to-duty (CTD) paradoxes, such as Chisholm paradoxes and its variants. This article uses an intuitionistic version of the ALC description logic, named iALC, to show how an ontology based on individually valid legal statements is able to avoid CTDs by providing models to them.
\end{abstract}

\section{Introduction}

Classical First Order Logic has been widely used as a basis for ontology creation and reasoning in many domains. These domains naturally include Legal Knowledge and Jurisprudence. As we expect, consistency is an important issue for legal ontologies. However, due to their inherently normative feature, coherence (consistency) in legal ontologies is more subtle than in other domains. Consistency, or absence of logical contradictions, seems more difficult to maintain when more than one law system can judge a case, what we call a conflict of laws. There are some legal mechanisms to solve these conflicts such as stating privileged fori or other ruling jurisdiction. In most of the cases, the conflict is solved by adopting a law hierarchy or precedence, rather better, ordering on laws. Even under these precedence mechanisms, coherence is still a major issue in legal systems. Each layer in this legal hierarchy has to be consistent. Since consistency is a direct consequence of how one deals with the logical negation, negation is also a main concern in legal systems. Deontic Logic, here considered as an extension of Classical Logic, has been widely used to formalize the normative aspects of the legal knowledge. There is some disagreement on using deontic logic, and any of its variants, to this task. Since a seminal paper by Alchourron and Martino (Alchourron \& Martino, 1990), the propositional aspect of laws has been under discussion. In (Alchourron \& Martino, 1990), the authors argue that laws are not to be considered as propositions, in full agreement with Hans Kelsen jurisprudence. The Kelsenian approach to Legal Ontologies considers the term "ontologies on laws" more appropriate than "law ontology". In previous works, we showed that Classical logic is not adequate to cope with a Kelsenian based Legal Ontology. Because of the popularity of Description Logic for expressing ontologies nowadays, we developed an Intuitionistic version of Description Logic particularly devised to express Legal Ontologies. This logic is called iALC. In this article, we show how iALC avoids some Contrary-to-duty paradoxes, as Chisholm paradox and other paradoxes that appear in deontic logic, such as the good samaritan and the knower. For these paradoxes, we provide iALC models. Finally, we discuss the main role of the intuitionistic negation in this issue, finding out that its success may be a consequence of its paracomplete logical

\footnotetext{
${ }^{1}$ Pontifícia Universidade Católica do Rio de Janeiro, Departamento de Informática. E-mail: edward.haeusler@gmail.com.

${ }^{2}$ Fundação Getúlio Vargas, Escola de Matemática Aplicada. E-mail: alexandre.rademaker@fgv.br. DOI : 10.21452/LnA_serie_n_v01_book_seminario-logica-no-aviao-2013-2018_hauesler-reademaker_p.44-59
} 
aspect. This investigation opens the use of other paracomplete logics in accomplishing a logical basis for Kelsenian legal ontologies, as a complementary solution to those based on paraconsistent logics, see (M.E. Coniglio, 2009).

\section{A brief discussion on Kelsenian Jurisprudence and its logic}

A very important task in jurisprudence (legal theory) is to make precise the use of the term "law", the individuation problem, and it is one of the most fundamental open questions in jurisprudence. It requires firstly answering the question "What is to count as one complete law?" (Raz, 1972). There are two main approaches to answer this question. One approach is to consider "the law" as the result of a natural process that yields a set of norms responsible for stating perfect social behavior. Another approach is to consider "the law" as a set of individual legal statements, each of them created to enforce a positively desired behavior in the society. As a consequence, in the first approach, the norms say what are the best morally speaking accepted state of affairs in a particular society, while, in the second approach, each legal statement rules an aspect of the society that the legislature wants to enforce the behavior. The first is more related to what is called Natural Law and the last to Legal positivism. We can say that the Legal positivism is closer to the way modeling is taken in Computer Science. In the natural approach to the law, it is even harder to define a system of laws than in the legal positivism. The natural approach demands stronger knowledge of the interdependency between the underlying legal statements than legal positivism. Because of that, the natural approach, in essence, is harder to be shared with practical jurisprudence principles, since they firstly are concerned to justify the law, on an essentially moral basis. This justification is quite hard to maintain from a practical point of view.

The coherence of "the law" in both approaches is essential. A debate on whether coherence is built-in by the restrictions induced by Nature in an evolutionary way, or whether coherence should be an object of knowledge management, seems to be a long debate. Despite that, legal positivism seems to be more suitable to Legal Artificial Intelligence. From the logical point of view, the natural approach is also harder to deal with than the positivist one. When describing a morally desired state-of-affairs, the logical statements take the form of propositions that has as a model best of the moral worlds. Deontic logic is suitable to be used to fulfill this task. However, a legal statement ("a law") is essentially an individual sentence that can also be seen as an order (mandatory command), and hence, it is not a proposition at all. As a consequence, deontic logic is not appropriate to be used in knowledge bases. Besides that, (Valente, 1995) shows that deontic logic does not properly distinguish between the normative status of a situation from the normative status of a norm (rule). We think that the best jurisprudence basis for Legal ontologies and reasoning is Legal positivism. Thus, we will be talking a legal ontology as an ontology about (individual) laws, and not an ontology on "the law".

Hans Kelsen initialized the Legal positivism tradition in 1934, for a contemporary reference see (Kelsen, 1991). He used this positive aspect of the legislature to define a theory of pure law and applied it to the problem of transfer citizen's rights and obligations from one country to other when crossing boarders. He produces a quite good understanding of what nowadays we denominate Private International Law. This achievement was so important that in many references on international law, Kelsen jurisprudence is the basis 
for discussions on conflict-of-laws derived from different statements coming from different fori. ${ }^{3}$

In what follows we introduce the main terminology and concepts of Kelsenian jurisprudence that we use in this article. We can summarize Kelsen theory of pure law in three principles:

(1) According to what was discussed above, individually valid legal statements are the first-class citizens of our ontology. Thus, only inhabitants of the Legal knowledge base are individual laws, see (Kelsen, 1967, supra note 5, pp 9-10) ${ }^{4}$. For example, if it is the case that Maria is married with John, and, this was legally celebrated, then "Maria-married-with-John" is an individually valid legal statement, and hence, it is a member of the Legal Ontology;

(2) Kelsen also says that that the validity of a legal norm can only be provided concerning the validity of another, and higher, one. So, $n_{1}$, a norm, is legally valid if, and only if, it was created or promulgated in agreement with other, and higher, legally valid norm, $n_{2}$. This justification induces a precedence relationship between norms that is transitive, that is, if $n_{1}$ precedes $n_{2}$, and, $n_{2}$ precedes $n_{3}$, then $n_{1}$ recedes $n_{3} ;{ }^{5}$

(3) There is a mechanism for relating laws from one Legal system to another, the socalled "choice-of-law rule". This mechanism is very important to the development of a concept of International Law. Assume that Mary-is-married-with-John is an individual legal statement in legal system $A$. Assume also that Mary is a citizen of a country adopting legal system $B$. Is there any legal statement in $B$ ensuring that Mary is married in $B$ ? Well, this depends on $B$ itself, but there is a way to connected the individual law Mary-is-married-with-John in $A$ to Mary-is-married-with-John in B. In some legal systems, this is accomplished by what Kelsen denominated "a connection". As shown in the following quotation from (Kelsen, 1946, page 247), the connection between the laws of $A$ and $B$ is made by reference, but, in fact, each law belongs to its respective legal system. In this specific case we can consider Mary-is-married-with-John in system $A$ is connected to Mary-is-married-with-John in legal system $B$ the connection Lex Loci Celebrationis.

... the law of one State prescribes the application of the law of another State, and the latter does not object or demand it. It has no right to do so since it is not really its own law which is applied by the other State. The latter applies norms of its own law. The fact that these norms have the same contents as corresponding norms of another State does not concern the latter...Since the specific technique of these norms consists in "referring" to the norms of another system and by so doing incorporating norms of identical contents into their own legal system, it would be more justifiable to call them "reference rules"... The reference

3 "It is one of Kelsen's frequently repeated doctrines that conflict of norms, in the absence of a normative procedure for resolving the conflict, shatters the concept of a unified system", is highly emphasized in Hughes (Hughes, 1971), for example, and it is one of the principles most cited when Kelsen jurisprudence is presented.

${ }^{4}$ Kelsen takes norms and valid norms as synonyms. To say that a legal norm is valid is to say that it exists, is affirmed by Kelsen

${ }^{5}$ See (Kelsen, 1967, supra note 5, p. 196-7). This can be also found in (Kelsen, 1946, supra note 5, p. 110-1) 
rule, that is ... the norm regulating the application of foreign law, may be distinguished from the norm to be applied, that is, the norm referred to. Only the former is a norm of private international law. But from a functional point of view, the one is essentially connected with the other.

Nowadays it is a common terminology in Private International Law the use of the connecting factors or legal connections between individual laws in a different legal system. Only to enumerate some of them: Lex-Domicilii, Lex-Patriae, Lex-loci-contratum, Lexloci-solutionis, etc.

There is a philosophical problem with the principle 2 above. It demands the existence of basic laws. These basic laws do not have their validity/existence as a consequence of other more basic laws. Kelsen name these basic laws Grundnorms. Their validity is based on legislature acts and in a certain sense is derived from the sovereign of the State. It is out of the scope of this article to discuss such problem in Kelsen's jurisprudence. We take as granted that Kelsen jurisprudence can adequately support most of the existent legal systems, a definitively not an unreal working hypothesis.

From the three principles above, we have some very simple ontological commitments:

I: Individuals are laws;

II: There is a transitive and reflexive relationship between individual laws that reflects the natural precedence relationship between laws;

III: There are legal connections between individual laws in different legal systems or between different fori in the same broader legal system.

From these commitments, we derive the basic constructs of the logic iALC. In the first place, our legal ontology relates concepts to legal systems. Description logics uses nominals to refer to individuals. So, an expression as $i: A$, stands for $i$ is an individual law, belonging to the legal system $A$, a concept.

From commitment we consider an expression as $i \preceq j$ standing for the individual law $i$ legally precedes individual law $j$. The subsumption relationship $A \subseteq B$, from description logic, denotes that $A$ is a legal subsystem of $B$. One could interpret this relation as the inclusion relationship. ${ }^{6}$ We discuss the implications of using negated contents together with Kelsenian jurisprudence in the following. This can be found in (Haeusler, de Paiva, \& Rademaker, 2011, 2010; Haeusler, Paiva, \& Rademaker, 2010; Haeusler, de Paiva, \& Rademaker, 2010) too.

Under the classical setting, a negated concept $\neg A$ denotes the set of all inhabitants of the domain that do not belong to the interpretation of $A$. Under ontological commitment there is no individual law that does not exist in, belong to, the domain. Since norms and laws are not propositions, it is a complete nonsense to negate a law. As we already seen, we can negate a concept on laws. Consider the collection of all Brazilian individual laws. Call it $B R$. In a classical setting $B R \sqcup \neg B R$ is the universe of laws. Thus, any law that it is not in $B R$ has to be a law outside $B R$, that is, belonging to $\neg B R$. For example, if Peter is 17 years old, it is not liable according to the Brazilian law. Is PETERIsLiable a valid law at all? If so, it has to belong to $\neg B R$. Using Kelsen in a classical setting, individual laws not belonging to a concept automatically belong to its complementary concept. The problem with this is that it is possible to create laws outside a jurisdiction or forum by the very simple act of considering or experimenting a legal situation. Nowadays in Brazil, the parliament is discussing the liability under the 16 years. By the simple fact of discussing

${ }^{6}$ In Classical ALC this is just the case, but we shown here that classical reasoning it is not a good choice for dealing with legal ontologies 
the validity of their corresponding individual laws, we are forced to accept they exist outside the Brazilian legal system. We do not consider this feature appropriate to legal ontology definition. Dealing with negations every time we assume the existence of a law may bring unnecessary complexity to legal ontology definition. Because the precedence relationship between laws, cf. ontological commitment, there is a natural alternative to classical logic, the intuitionistic logic (IL). According to IL semantics, $i: \neg A$, iff, for each law $j$, such that $i \preceq j$, it is not the case that $j: A$. This semantics means that $i$ does not provide any legal support for any individual law belong to $A$, which agrees with Kelsen jurisprudence on the hierarchy of individual laws.

Commitment gives rise to expressions of the form $m$ LexLociCelebrationis $m$, where $m$ is MARYIsMarriedWithJohn and LexLociCELEBrationis is a legal connection. Thus, if Abroad is the concept that represents all laws in Portugal, then the concept $\exists$ LexLociCelebrationis Portugal represents the Brazilian individual laws stating that Portuguese marriage is valid in Brazil. The private international law of any country is a collection of laws stated in similar ways for every possible legal connection. In (Haeusler, de Paiva, \& Rademaker, 2010) it is shown in detail a judicial case deriving that a renting contract is solving a conflict of laws in space through private international law.

\section{Some philosophical discussion on using Kelsen in legal ontologies}

We base our work on two ontological criteria: ${ }^{7}$

- Ontological Commitment (due to W.Quine), our logical approach is ontologically committed to Valid Legal Statements only, as discussed in section . The only nominals occurring in our logic language are valid individual laws, and;

- Ontological Parsimony, which is strongly related to Quine's ontological commitment too, with a mention of its stronger version also known as Occam's Razor, here denoted as OR.

The second criteria is based on: "One 'easy' case where OR can be straightforwardly applied is when a theory T, postulates entities which are explanatorily idle. Excising these entities from $\mathrm{T}$ produces a second theory, $\mathrm{T}^{*}$, which has the same theoretical virtues as $\mathrm{T}$ but a smaller set of ontological commitments. Hence, according to OR, it is rational to pick T* over T."

We observe that nominals, representing individuals, denote only valid individual laws and nothing in the iALC language described in the following section, is committed with non-valid individual laws, according to the second ontological criterion above, we do not have to consider non-valid individual laws. Technically speaking there is no element in the iALC language able to denote an invalid individual law in any model of any iALC theory. If something is a valid individual law regarded some legal system in some place in the world, then this individual belongs to our semantic universe.

This philosophical basis allows us to have only sets of valid individuals as semantics for iALC theories. Thus, as the a reviewer have already observed, this implies that $\neg A$ is the set of individual laws holding outside Brazil, and the classical negation is not adequate to denote this set. If we get $\neg A$ meaning "individual laws that do not hold in Brazil", the set of laws being a proper subset of the universe, and $A$ is the conjunctive property "laws + holds in $A$ ". Then the complement, $\neg A$ would be all elements of the universe which are

7 see Quine's "On What there is" article and http://plato.stanford.edu/entries/simplicity, for example to a primer ontological criteria 
either not a valid individual law or do not hold in Brazil. But there is no way to take the semantics in this way, for the semantics we get from the ontological commitment from section is given by "The individual valid laws holding outside of Brazil".

Finally, concerning contradictory individual laws, they can coexist in the same universe, since they are there because they hold in distinct legal systems. In fact they are apparently contradictory. For example, "There is death penalty" and "Death sentence is not allowed" can coexist, since there are countries where each of these legal statement are valid. Concretely: "There is death penalty":Iran and "Death sentence is not allowed":Brazil.

\section{The Logic iALC}

Classical Description Logic has been widely used as a basis for ontology creation and reasoning in many knowledge specific domains, including Legal AI.

An adequate intuitionistic semantics for negation in a legal domain comes to the fore when we take legally valid individual statements as the inhabitants of our legal ontology. This allows us to elegantly deal with particular situations of legal coherence, such as conflict of laws, as those solved by Private International Law analysis. In (Haeusler, de Paiva, \& Rademaker, 2010, 2010; Haeusler, Paiva, \& Rademaker, 2010) we present an Intuitionistic Description Logic, called iALC for Intuitionistic ALC (for Attributive Language with Complements, the canonical classical description logic system). A labeled sequent calculus for iALC based on a labeled sequent calculus for ALC (Rademaker, 2012), was also presented. In these previous articles, we discussed the jurisprudence foundation of our system, and show how we can perform a coherence analysis of "Conflict of Laws in Space" by means of iALC. This conflict happens when several laws can be applied, with different outcomes, to a case depending on the place where the case occurs. Typical examples are those ruling the rights of a citizen abroad.

In (Haeusler, de Paiva, \& Rademaker, 2010), the semantics of iALC is precisely provided and follows the framework for constructive modal logics presented by Simpson (Simpson, 1993) and adapted to description languages by Paiva (de Paiva, 2006). In the cited reference, we applied iALC to the problem of formalizing legal knowledge.

Description Logics are an important knowledge representation formalism, unifying and giving a logical basis to the well known AI frame-based systems of the eighties. Description logics are very popular right now. Given the existent and proposed applications of the Semantic Web, there has been a fair amount of work into finding the most well-behaved system of description logic that has the broadest application, for any specific domain. Description logics tend to come in families of logical systems, depending on which concept constructors you allow in the logic. Since description logics came into existence as fragments of first-order logic chosen to find the best trade-off possible between expressiveness and tractability of the fragment, several systems were discussed and in the taxonomy of systems that emerged the ALC has come to be known as the canonical one. The basic building blocks of description logics are concepts, roles and individuals. Concepts are described as unary predicates in usual first-order logic and roles as binary atomic predicates used to modify the concepts.

As discussed in (de Paiva, 2006), considering versions of constructive description logics makes sense, both from a theoretical and from a practical viewpoint. There are several possible and sensible ways of defining constructive description logics, whether your motivation is natural language semantics, (de Paiva, 2006), or Legal AI, (Haeusler, de Paiva, 
\& Rademaker, 2010). As far as constructive description logics are concerned, Mendler and Scheele have worked out a very compelling system cALC (Mendler \& Scheele, 2010), based on the constructive modal logic CK (Bellin, de Paiva, \& Ritter, 2001)), one possible choice for us. However in this note we follow a different path and describe a constructive version of ALC, based on the framework for constructive modal logics developed by Simpson (the system IK) in his phd thesis (Simpson, 1993) (For a proof-theoretic comparison between the constructive modal logics CK and IK one can see (Ranalter, 2010)).

Our motivation, besides Simpson's work, is the framework developed by Braüner and de Paiva in (Braüner \& de Paiva, 2006) for constructive Hybrid Logics. We reason that having already frameworks for constructive modal and constructive hybrid logics in the labelled style of Simpson, we might end up with the best style of constructive description logics, in terms of both solid foundations and ease of implementation. Since submitting this paper we have been told about the master thesis of Clément (Clément, 2008) which follows broadly similar lines. Clément proves soundness and completeness of this system and then provides a focused version of it, a very interesting development, as focused systems are, apparently, very useful for proof search.

Building up from the Simpson's constructive modal logics in (Braüner \& de Paiva, 2006) (called here IML), it is introduced intuitionistic hybrid logics, denoted by IHL. Hybrid logics add to usual modal logics a new kind of propositional symbols, the nominals, and also the so-called satisfaction operators. A nominal is assumed to be true at exactly one world, so a nominal can be considered the name of a world. If $x$ is a nominal and $X$ is an arbitrary formula, then a new formula $x: X$ called a satisfaction statement can be formed. The satisfaction statement $x: X$ expresses that the formula $X$ is true at one particular world, namely the world denoted by $x$. In hindsight one can see that IML shares with hybrid formalisms the idea of making the possible-world semantics part of the deductive system. While IML makes the relationship between worlds (e.g., $x R y)$ part of the deductive system, IHL goes one step further and sees the worlds themselves $x, y$ as part of the deductive system, (as they are now nominals) and the satisfaction relation itself as part of the deductive system, as it is now a syntactic operator, with modality-like properties.

Our Sequent Calculus for iALC was first presented in (de Paiva, Hausler, \& Rademaker, 2010) where we briefly described the immediate properties of this system and most importantly we discuss a case study of the use of iALC in legal AI.

A very important observation is that this article corrects and extends the presentation of iALC appearing in all previous articles. It points out the difference between iALC and the intuitionistic hybrid logic presented in (de Paiva, 2006). Completeness and soundness proofs are revised. A discussion on the computational complexity of iALC is also taken.

\section{Intuitionistic ALC}

The iALC logic is based on the framework for intuitionistic modal logic IK proposed in (Simpson, 1993; Fischer-Servi, 1984; Plotkin \& Stirling, 1986). These modal logics arise from interpreting the usual possible worlds definitions in an intuitionistic meta-theory. As we will see in the following paragraphs, ideas from (Braüner \& de Paiva, 2006) were also used, where the framework IHL, for intuitionistic hybrid logics, is introduced. iALC concepts are described as:

$$
C, D::=A|\perp| \top|\neg C| C \sqcap D|C \sqcup D| C \sqsubseteq D|\exists R . C| \forall R . C
$$


where $C, D$ stands for concepts, $A$ for an atomic concept, $R$ for an atomic role. We could have used distinct symbols for subsumption of concepts and the subsumption concept constructor but this would blow-up the calculus presentation. This syntax is more general than standard ALC since it includes subsumption $\sqsubseteq$ as a concept-forming operator. We have no use for nested subsumptions, but they do make the system easier to define, so we keep the general rules. Negation could be defined via subsumption, that is, $\neg C=C \sqsubseteq \perp$, but we find it convenient to keep it in the language. The constant $T$ could also be omitted since it can be represented as $\neg \perp$.

A constructive interpretation of iALC is a structure $\mathcal{I}$ consisting of a non-empty set $\Delta^{\mathcal{I}}$ of entities in which each entity represents a partially defined individual; a refinement preordering $\preceq^{\mathcal{I}}$ on $\Delta^{\mathcal{I}}$, i.e., a reflexive and transitive relation; and an interpretation function . ${ }^{\mathcal{I}}$ mapping each role name $R$ to a binary relation $R^{\mathcal{I}} \subseteq \Delta^{\mathcal{I}} \times \Delta^{\mathcal{I}}$ and atomic concept $A$ to a set $A^{\mathcal{I}} \subseteq \Delta^{\mathcal{I}}$ which is closed under refinement, i.e., $x \in A^{\mathcal{I}}$ and $x \preceq^{\mathcal{I}} y$ implies $y \in A^{\mathcal{I}}$. The interpretation $\mathcal{I}$ is lifted from atomic concepts to arbitrary concepts via:

$$
\begin{aligned}
\top^{\mathcal{I}} & ={ }_{d f} \Delta^{\mathcal{I}} \\
\perp^{\mathcal{I}} & ={ }_{d f} \emptyset \\
(\neg C)^{\mathcal{I}} & ={ }_{d f}\left\{x \mid \forall y \in \Delta^{\mathcal{I}} . x \preceq y \Rightarrow y \notin C^{\mathcal{I}}\right\} \\
(C \sqcap D)^{\mathcal{I}} & ={ }_{d f} C^{\mathcal{I}} \cap D^{\mathcal{I}} \\
(C \sqcup D)^{\mathcal{I}} & ={ }_{d f} C^{\mathcal{I}} \cup D^{\mathcal{I}} \\
(C \sqsubseteq D)^{\mathcal{I}} & ={ }_{d f}\left\{x \mid \forall y \in \Delta^{\mathcal{I}} \cdot\left(x \preceq y \text { and } y \in C^{\mathcal{I}}\right) \Rightarrow y \in D^{\mathcal{I}}\right\} \\
(\exists R . C)^{\mathcal{I}} & ={ }_{d f}\left\{x \mid \exists y \in \Delta^{\mathcal{I}} \cdot(x, y) \in R^{\mathcal{I}} \text { and } y \in C^{\mathcal{I}}\right\} \\
(\forall R . C)^{\mathcal{I}} & ={ }_{d f}\left\{x \mid \forall y \in \Delta^{\mathcal{I}} . x \preceq y \Rightarrow \forall z \in \Delta^{\mathcal{I}} \cdot(y, z) \in R^{\mathcal{I}} \Rightarrow z \in C^{\mathcal{I}}\right\}
\end{aligned}
$$

Following the semantics of IK, the structures $\mathcal{I}$ are models for iALC if they satisfy two frame conditions:

F1: if $w \leq w^{\prime}$ and $w R v$ then $\exists v^{\prime} \cdot w^{\prime} R v^{\prime}$ and $v \leq v^{\prime}$

F2: if $v \leq v^{\prime}$ and $w R v$ then $\exists w^{\prime} \cdot w^{\prime} R v^{\prime}$ and $w \leq w^{\prime}$

The above conditions are diagrammatically expressed as:

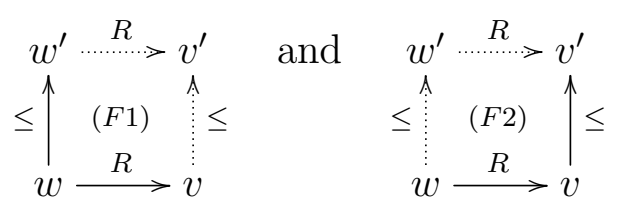

Our setting simplifies (Mendler \& Scheele, 2010), since $i \mathcal{A L C}$ satisfies (like classical $\mathcal{A L C}) \exists R . \perp=\perp$ and $\exists R .(C \sqcup D)=\exists R . C \sqcup \exists R . D$.

In contrast with the above mentioned approaches, ours assign a truth values to some formulas, also called assertions, they are not concepts as in (Braüner \& de Paiva, 2006), for example. Below we define the syntax of general assertions $(A)$ and nominal assertions $(N)$ for ABOX reasoning in iALC. Formulas $(F)$ also includes subsumption of concepts interpreted as propositional statements.

$$
N::=x: C|x: N \quad A::=N| x R y \quad F::=A \mid C \sqsubseteq C
$$

where $x$ and $y$ are nominals, $R$ is a role symbol and $C$ is a concept. In particular, this allows $x:(y: C)$, which is a perfectly valid nominal assertion.

DEFINITION 1. (outer nominal) In a nominal assertion $x: \gamma, x$ is said to be the outer nominal of this assertion. That is, in an assertion of the form $x:(y: \gamma), x$ is the outer nominal. 
We write $\mathcal{I}, w \models C$ to abbreviate $w \in C^{\mathcal{I}}$ which means that entity $w$ satisfies concept $C$ in the interpretation $\mathcal{I}^{8}$. Further, $\mathcal{I}$ is a model of $C$, written $\mathcal{I} \models C$ iff $\forall w \in \mathcal{I}$, we have that $\mathcal{I}, w \models C$. Finally, $\models C$ means $\forall \mathcal{I}$, we have that $\mathcal{I} \models C$. All previous notions are extended to sets $\Phi$ of concepts in the usual universal fashion. Given the hybrid satisfaction statements, the interpretation and semantic satisfaction relation are extended in the expected way. The statement $\mathcal{I}, w=x: C$ holds, if and only if, $\forall z_{x} \succeq^{\mathcal{I}}$ $x$, we have that, $\mathcal{I}, z_{x} \models C$. In a similar fashion, $\mathcal{I}, w \models x R y$ holds ,if and only if, $\forall z_{x} \succeq x . \forall z_{y} \succeq y .\left(x_{x}^{\mathcal{I}}, z_{y}^{\mathcal{I}}\right) \in R^{\mathcal{I}}$. That is, the evaluation of the hybrid formulas does not take into account only the world $w$, but it has to be monotonically preserved. It can be observed that for every $w^{\prime}$, if $x^{\mathcal{I}} \preceq w^{\prime}$ and $\mathcal{I}, x^{\prime} \models \alpha$, then $\mathcal{I}, w^{\prime} \models \alpha$ is a property holding on this satisfaction relation.

In common reasoning tasks the interpretation $\mathcal{I}$ and the entity $w$ in a verification goal such as $\mathcal{I}, w \models \delta$ are not given directly but are themselves axiomatized by sets of concepts and formulas. Usually we have a set $\Theta{ }^{9}$ of formulas and the set $\Gamma$ of concepts. Accordingly:

Definition 2. We write $\Theta, \Gamma \models \delta$ if it is the case that:

$$
\forall \mathcal{I} \text {. if }\left(\forall x \in \Delta^{\mathcal{I}} .(\mathcal{I}, x \models \Theta) \text {, then } \forall(N o m(\Gamma, \delta)) . \forall \vec{z} \succeq N o m(\Gamma, \delta) .(\mathcal{I}, \vec{z} \models \Gamma \Rightarrow \mathcal{I}, \vec{z} \models \delta)\right.
$$

where $\vec{z}$ denotes a vector of variables $z_{1}, \ldots, z_{k}$ and $N o m(\Gamma, \delta)$ is the vector of all outer nominals occurring in each nominal assertion of $\Gamma \cup\{\delta\} . x$ is the only outer nominal of a nominal assertion $\{x: \gamma\}$, while a (pure) concept $\gamma$ has no outer nominal.

A Hilbert calculus for iALC is provided following (Plotkin \& Stirling, 1986; Simpson, 1993; Fischer-Servi, 1984). It consists of all axioms of intuitionistic propositional logic plus the axioms and rules displayed in Figure 1. The Hilbert calculus implements TBoxreasoning. That is, it decides the semantical relationship $\Theta, \emptyset \models C$. $\Theta$ has only formulas as members.

0. all substitution instances of theorems of IPL

1. $\forall R .(C \sqsubseteq D) \sqsubseteq(\forall R . C \sqsubseteq \forall R . D)$

2. $\exists R .(C \sqsubseteq D) \sqsubseteq(\exists R . C \sqsubseteq \exists R . D)$

3. $\exists R .(C \sqcup D) \sqsubseteq(\exists R . C \sqcup \exists R . D)$

4. $\exists R \cdot \perp \sqsubseteq \perp$

5. $(\exists R . C \sqsubseteq \forall R . C) \sqsubseteq \forall R .(C \sqsubseteq D)$

MP If $C$ and $C \sqsubseteq D$ are theorems, $D$ is a theorem too.

$\mathrm{Nec}$ If $C$ is a theorem then $\forall R . C$ is a theorem too.

FIGURE 1. The iALC axiomatization

A Sequent Calculus for iALC is also provided. The logical rules of the Sequent Calculus for iALC are presented in Figure 2. ${ }^{10}$ The structural rules and the cut rule are omitted but they are as usual. The $\delta$ stands for concepts or assertions $(x: C$ or $x R y), \alpha$ and $\beta$

\footnotetext{
${ }^{8}$ In IHL, this $w$ is a world and this satisfaction relation is possible world semantics

${ }^{9}$ Here we consider only acycled TBox with $\sqsubseteq$ and $\equiv$.

10 The reader may want to read Proof Theory books, for example, (Takeuti, 2013; Buss, 1998; Negri \& Von Plato, 2008; Girard, Taylor, \& Lafont, 1989).
} 
for concept and $R$ for role. $\Delta$ is a set of formulas. In rules p- $\exists$ and p- $\forall$, the syntax $\forall R . \Delta$ means $\{\forall R . \alpha \mid \alpha \in$ concepts $(\Delta)\}$, that is, all concepts in $\Delta$ are universal quantified with the same role. The assertions in $\Delta$ are kept unmodified. In the same way, in rule $\mathrm{p}-\mathrm{N}$ the addition of the nominal is made only in the concepts of $\Delta$ (and in $\delta$ if that is a concept) keeping the assertions unmodified.

The propositional connectives $(\sqcap, \sqcup, \sqsubseteq)$ rules are as usual, the rule $\sqcup_{2}$-r is omitted. The rules are presented without nominals but for each of these rules there is a counterpart with nominals. For example, the rule -r has one similar:

$$
\frac{\Delta, x: \alpha \Rightarrow x: \beta}{\Delta \Rightarrow x:(\alpha \sqsubseteq \beta)} \mathrm{n}-\sqsubseteq-\mathrm{r}
$$

The main modification comes for the modal rules, which are now role quantification rules. We must keep the intuitionistic constraints for modal operators. Rule $\exists$-l has the usual condition that $y$ is not in the conclusion. Concerning the usual condition on the $\forall$-r rule, it is not the case in this system, for the interpretation of the a nominal assertion in a sequent is already implicitly universal (Definition 2).

$$
\begin{gathered}
\overline{\Delta, \delta \Rightarrow \delta} \\
\frac{\Delta, x R y \Rightarrow y: \alpha}{\Delta \Rightarrow x: \forall R . \alpha} \forall-\mathrm{r} \\
\frac{\Delta \Rightarrow x R y \quad \Delta \Rightarrow y: \alpha}{\Delta \Rightarrow x: \exists R . \alpha} \exists-\mathrm{r} \\
\frac{\Delta, \alpha \Rightarrow \beta}{\Delta \Rightarrow \alpha \sqsubseteq \beta} \sqsubseteq-\mathrm{r} \\
\frac{\Delta \Rightarrow \alpha \Rightarrow \beta}{\Delta \Rightarrow \alpha \sqcap \beta} \sqcap-\mathrm{r} \\
\frac{\Delta \Rightarrow \alpha}{\Delta \Rightarrow \alpha \sqcup \beta} \sqcup_{1}-\mathrm{r} \\
\frac{\Delta, \alpha \Rightarrow \beta}{\forall R . \Delta, \exists R . \alpha \Rightarrow \exists R . \beta} \mathrm{p}-\exists \\
\frac{\Delta \Rightarrow \delta}{x: \Delta \Rightarrow x: \delta} \mathrm{p}-\mathrm{N}
\end{gathered}
$$

FIgure 2. The System $\mathrm{SC}_{\mathrm{iALC}}$ : logical rules

THEOREM 1. The sequent calculus described in Fig. 2 is sound and complete for TBox reasoning, that is $\Theta, \emptyset \models C$ if and only if $\Theta \Rightarrow C$ is derivable with the rules of Figure 2.

The completeness of our system is proved relative to the axiomatization of iALC, shown in Figure 1. The proof is presented in Section .

The soundness of the system is proved directly from the semantics of iALC including the ABOX, that is, including nominals. The semantics of a sequent is defined by the satisfaction relation, as shown in Definition 2. The sequent $\Theta, \Gamma \Rightarrow \delta$ is valid if and only 
if $\Theta, \Gamma \models \gamma$. Soundness is proved by showing that each sequent rule preserves the validity of the sequent and that the initial sequent is valid. This proof is presented in Section .

We note that although we have here fixed some inaccuracies in the presentation of the iALC semantics in (de Paiva et al., 2010), the system presented here is basically the same, excepted that here the propositional rules are presented without nominals. Given that, the soundness of the system proved in (de Paiva et al., 2010) can be still considered valid without further problems. Note also that the proof of soundness provides in Section is regarded the full language of iALC. It considers nominals and assertion on nominals relationship, that is it concerns $\mathrm{ABOX}$ and TBOX. The proof of completeness is for the TBOX only. A proof of completeness for ABOX can be done by the method of canonical models. For the purposes of this article, we choose to show the relative completeness proof with the sake of showing a simpler proof concerning TBOX.

\section{The completeness of $\mathrm{SC}_{\mathrm{iALC}}$ system}

We show the relative completeness of $\mathrm{SC}_{\mathrm{iALC}}$ regarding the axiomatic presentation of iALC presented in Figure 1. To prove the completeness of $\mathrm{SC}_{\mathrm{iALC}}$ it is sufficient to derive in $\mathrm{SC}_{\mathrm{iALC}}$ the axioms $1-5$ of iALC. It is clear that all substitution instances of IPL theorems can also be proved in $\mathrm{SC}_{\mathrm{iALC}}$ using only propositional rules. The MP rule is a derived rule from the $\mathrm{SC}_{\mathrm{iALC}}$ using the cut rule. The Nec rule is the $\mathrm{p}-\forall$ rule in the system with $\Delta$ empty. In the first two proofs below do not use nominals for given better intuition of the reader about the use of rules with and without nominals.

Axiom 1:

Axiom 2:

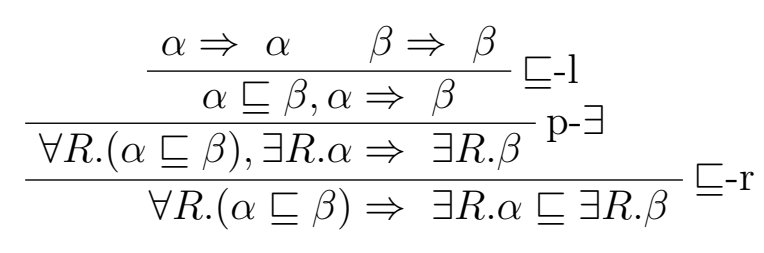

Axiom 3:

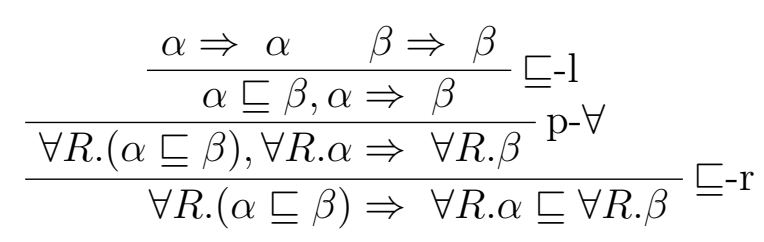

Axiom 4:

$$
\frac{\frac{x R y, y: \perp \Rightarrow x: \perp}{x: \exists R . \perp \Rightarrow x: \perp} \exists-1}{\Rightarrow x:(\exists R \cdot \perp \sqsubseteq \perp)} \sqsubseteq-\mathrm{r}
$$

Axiom 5:

$$
\frac{\frac{x: \exists R . \alpha \Rightarrow x: \exists R . \alpha}{x: \exists R . \alpha \Rightarrow x:(\exists R . \alpha \sqcup \exists R . \beta)} \sqcup_{1}-\mathrm{r} \quad \frac{x: \exists R . \beta \Rightarrow x: \exists R . \beta}{x: \exists R . \beta \Rightarrow x:(\exists R . \alpha \sqcup \exists R . \beta)} \sqcup_{2}-\mathrm{r}}{x: \exists R .(\alpha \sqcup \beta) \Rightarrow x:(\exists R . \alpha \sqcup \exists R . \beta)}
$$

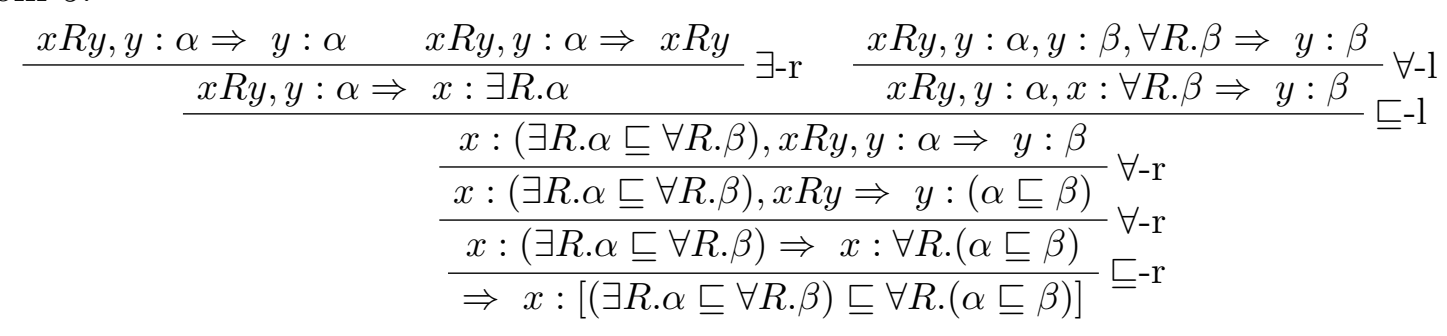




\section{Soundness of $\mathrm{SC}_{\mathrm{iALC}}$ system}

In this section we prove that.

Proposition 1. If $\Theta, \Gamma \Rightarrow \delta$ is provable in $S C_{i A L C}$ then $\Theta, \Gamma \models \gamma$.

Proof: We prove that each sequent rule preserves the validity of the sequent and that the initial sequents are valid. The definition of a valid sequent $(\Theta, \Gamma \models \gamma)$ is presented in Definition 2.

The validity of the axioms is trivial. We first observe that any application of the rules $\sqsubseteq-\mathrm{r}, \sqsubseteq-1, \sqcap-\mathrm{r}, \sqcap-\mathrm{l}, \sqcup_{1}-\mathrm{r}, \sqcup_{2}-\mathrm{r}, \sqcup-\mathrm{l}$ of $\mathrm{SC}_{\mathrm{iALC}}$ where the sequents do not have any nominal, neither in $\Theta$ nor in $\Gamma$, is sound regarded intuitionistic propositional logic Kripke semantics, to which the validity definition above collapses whenever there is no nominal in the sequents. Thus, in this proof we concentrate in the case where there are nominals. We first observe that the nominal version of $\sqsubseteq$-r, the validity of the premises includes

$$
\forall(\operatorname{Nom}(\Gamma, \delta)) . \forall \vec{z} \succeq \operatorname{Nom}(\Gamma, \delta) .(\mathcal{I}, \vec{z}=\Gamma \Rightarrow \mathcal{I}, \vec{z} \models \delta)
$$

This means that $\Gamma$ holds in any worlds $\vec{z} \succeq \vec{x}$ for the vector $\vec{x}$ of nominals occurring in $\Gamma$. This includes the outer nominal $x_{i}$ in $\delta$ (if any). In this case the semantics of $\sqsubseteq$ is preserved, since $\vec{z}$ includes $z_{i} \succeq x_{i}$. With the sake of a more detailed analysis, we consider the following instance:

$$
\frac{x: \alpha_{1}, y: \alpha_{2} \Rightarrow x: \beta}{\alpha_{1} \Rightarrow x: \alpha_{2} \sqsubseteq \beta} \sqsubseteq-\mathrm{r}
$$

Consider an iALC structure $\mathcal{I}=\left\langle\mathcal{U}, \preceq, R^{\mathcal{I}} \ldots, C^{\mathcal{I}}\right\rangle$ In this case, for any $\mathcal{I}$ and any $z_{1}, z_{2} \in \mathcal{U}^{\mathcal{I}}$ if $z_{1} \succeq x^{\mathcal{I}}, z_{1} \succeq y^{\mathcal{I}}$, such that, $\mathcal{I}, z_{i} \models \alpha_{1}$ and $\mathcal{I}, z_{i} \models \alpha_{2}$, we have that $\mathcal{I}, z_{i} \models x: \beta$, since the premise is valid, by hypothesis. In this case, by the semantics of $\sqsubseteq$ we have $\mathcal{I}, z_{i} \models x: \alpha_{1} \sqsubseteq \beta$. The conclusion of the rule is valid too.

The argument shown above for the $\sqsubseteq$-r rule is analogous for the nominal versions of

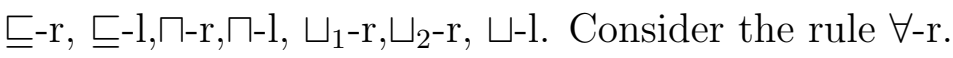

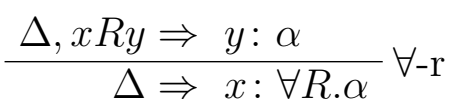

Since the premise is valid we have that if $\forall z_{x} \succeq x^{\mathcal{I}}, \forall z_{y} \succeq y^{\mathcal{I}},\left(z_{x}, z_{y}\right) \in R^{\mathcal{I}}$ then $\forall z_{y} \succeq y^{\mathcal{I}} \cdot \mathcal{I}, z_{y} \models \gamma$. This entails that $x^{\mathcal{I}} \in(\forall R \cdot \gamma)^{\mathcal{I}}$, for $x^{\mathcal{I}} \succeq x^{\mathcal{I}}$. We observe that by the restriction on the rule application, $y$ does not occur in $\Delta$, it only occurs in $x R y$ and $y: \alpha$. The truth of these formulas are subsumed by $\forall R . \gamma$. The conclusion does not need to consider them any more. The conclusion is valid too. Another way to see its soundness is to prove that if $x R y \Rightarrow y: \alpha$ is valid, then so is $\Rightarrow x: \forall R . \alpha$. This can be show by the following reasoning:

$$
\forall x^{\mathcal{I}} \forall y^{\mathcal{I}} \forall z_{x} \forall z_{y}\left(z_{x} \succeq x^{\mathcal{I}} \rightarrow\left(z_{y} \succeq y^{\mathcal{I}} \rightarrow\left(\left(z_{x}, z_{y}\right) \in R^{\mathcal{I}} \rightarrow \mathcal{I}, z_{y} \models y: \alpha\right)\right)\right)
$$

that is the same as:

$$
\forall x^{\mathcal{I}} \forall y^{\mathcal{I}} \forall z_{x} \forall z_{y}\left(z_{x} \succeq x^{\mathcal{I}} \rightarrow\left(z_{y} \succeq y^{\mathcal{I}} \rightarrow\left(\left(z_{x}, z_{y}\right) \in R^{\mathcal{I}} \rightarrow \mathcal{I}, y^{\mathcal{I}}=\alpha\right)\right)\right)
$$

Using the fact that $\forall y^{\mathcal{I}}\left(y^{\mathcal{I}} \succeq y^{\mathcal{I}}\right)$, we obtain:

$$
\forall x^{\mathcal{I}} \forall z_{x}\left(z_{x} \succeq x^{\mathcal{I}} \rightarrow \forall y^{\mathcal{I}}\left(\left(z_{x}, y^{\mathcal{I}}\right) \in R^{\mathcal{I}} \rightarrow \mathcal{I}, y^{\mathcal{I}} \models \alpha\right)\right)
$$

The above condition states that $\Rightarrow x: \forall R . \alpha$ is valid.

$$
\forall x^{\mathcal{I}} \forall y^{\mathcal{I}} \forall z_{x} \forall z_{y}\left(z_{x} \succeq x^{\mathcal{I}} \rightarrow\left(z_{y} \succeq y^{\mathcal{I}} \rightarrow\left(\left(z_{x}, z_{y}\right) \in R^{\mathcal{I}} \rightarrow \mathcal{I}, z_{y} \models y: \alpha\right)\right)\right)
$$

Consider the rule $\forall$-l: 


$$
\frac{\Delta, x: \forall R . \alpha, y: \alpha, x R y \Rightarrow \delta}{\Delta, x: \forall R . \alpha, x R y \Rightarrow \delta} \forall-1
$$

As in the $\forall$-r case, we analyze the simplest validity preservation: if $x: \forall R . \alpha \wedge x R y$ is valid, then so is $x: \forall R . \alpha \wedge y: \alpha \wedge x R y$. The first condition is:

$$
\begin{aligned}
& \forall x^{\mathcal{I}} \forall y^{\mathcal{I}} \forall z_{x}\left(z _ { x } \succeq x ^ { \mathcal { I } } \rightarrow \forall z _ { y } \left(z_{y} \succeq y^{\mathcal{I}} \rightarrow\right.\right. \\
& \qquad\left(\left(\mathcal{I}, z_{y}=x: \forall R . \alpha\right) \wedge\left(\mathcal{I}, z_{y}=x: \forall R . \alpha\right) \wedge\left(\left(z_{x}, z_{y}\right) \in R^{\mathcal{I}}\right) \rightarrow\right. \\
& \left.\left.\left.\quad\left(\mathcal{I}, z_{y} \models y: \alpha\right) \wedge\left(\mathcal{I}, z_{x} \models y: \alpha\right)\right)\right)\right)
\end{aligned}
$$

Using $z_{y}=y^{\mathcal{I}}$, eliminating $z_{x}$ from the term, and, using the fact that $\mathcal{I}, z_{y} \models y: \alpha$ is valid, iff, $\mathcal{I}, y^{\mathcal{I}}=\alpha$, we obtain

$$
\begin{aligned}
& \forall x^{\mathcal{I}} \forall y^{\mathcal{I}} \forall z_{x}\left(z _ { x } \succeq x ^ { \mathcal { I } } \rightarrow \forall z _ { y } \left(z_{y} \succeq y^{\mathcal{I}} \rightarrow\right.\right. \\
& \left.\left.\quad\left(\left(\mathcal{I}, z_{y}=x: \forall R . \alpha\right) \wedge\left(\mathcal{I}, z_{y}=x: \forall R . \alpha\right) \wedge\left(\left(z_{x}, z_{y}\right) \in R^{\mathcal{I}}\right) \rightarrow(\mathcal{I}, y \models \alpha)\right)\right)\right)
\end{aligned}
$$

Consider the semantics of $\exists R . \alpha$ :

$$
(\exists R . \alpha)^{\mathcal{I}}=_{d f}\left\{x \mid \exists y \in \mathcal{U}^{\mathcal{I}} .(x, y) \in R^{\mathcal{I}} \text { and } y \in \alpha^{\mathcal{I}}\right\}
$$

and the following rule:

$$
\frac{\Delta \Rightarrow x R y \quad \Delta \Rightarrow y: \alpha}{\Delta \Rightarrow x: \exists R . \alpha} \exists-\mathrm{r}
$$

We can see that the premises of the rule entails the conclusion. The premises correspond to the following conditions:

$$
\forall x^{\mathcal{I}} \forall y^{\mathcal{I}} \forall z_{x}\left(z_{x} \succeq x^{\mathcal{I}} \rightarrow \forall z_{y}\left(z_{y} \succeq y^{\mathcal{I}} \rightarrow\left(\left(z_{x}, z_{y}\right) \in R^{\mathcal{I}}\right)\right)\right)
$$

and

$$
\forall y^{\mathcal{I}} \forall z_{y}\left(z_{y} \succeq y^{\mathcal{I}} \rightarrow\left(\left(\mathcal{I}, z_{y} \models y: \alpha\right)\right)\right)
$$

Instantiating in both conditions $z_{y}=y^{\mathcal{I}}$ and $z_{x}=x^{\mathcal{I}}$, this yields $\left(x^{\mathcal{I}}, y^{\mathcal{I}}\right) \in R^{\mathcal{I}}$, such that $\mathcal{I}, y^{\mathcal{I}} \models \alpha$, so $\mathcal{I}, z_{x} \models x^{\mathcal{I}}: \exists R$. $\alpha$. Thus, $\exists$-r is sound. The soundness of $\exists$-l is analogous to $\forall-l$.

Finally, it is worth noting that, for each rule, we can derive the soundness of its nonnominal version from the proof of soundness of its nominal version. For instance, the soundness of the nominal version of rule $\sqcup-\mathrm{l}$ depends on the diamond conditions $\mathrm{F} 1$ and F2. The soundness of its non-nomimal version, is a consequence of the soundness of the nominal version.

The rules below have their soundness proved as a consequence of the following reasonings in first-order intuitionistic logic that are used for deriving the semantics of the conclusions from the semantics of the premises:

$$
\begin{aligned}
& (\mathbf{p}-\exists): \forall x(A(x) \wedge B(x) \rightarrow C(x)) \models \forall x A(x) \wedge \exists x B(x) \rightarrow \exists x C(x) ; \\
& (\mathbf{p}-\forall):(A(x) \models B(x)) \text { implies } \forall y(R(y, x) \rightarrow A(x)) \models \forall y(R(y, x) \rightarrow B(x)) ; \\
& (\mathbf{p}-\mathbf{N}): \text { if } A \models B \text { then for every Kripke model } \mathcal{I} \text { and world } x^{\mathcal{I}} \text {, if } \mathcal{I}, x^{\mathcal{I}} \models A \text { then } \\
& \mathcal{I}, x^{\mathcal{I}} \models B . \\
& \Delta, \alpha \Rightarrow \beta \\
& \frac{\Delta R . \Delta, \exists R . \alpha \Rightarrow \exists R . \beta}{} \mathrm{p}-\exists \frac{\Delta \Rightarrow \alpha}{\forall R . \Delta \Rightarrow \forall R . \alpha} \mathrm{p}-\forall \frac{\Delta \Rightarrow \delta}{x: \Delta \Rightarrow x: \delta} \mathrm{p}-\mathrm{N}
\end{aligned}
$$




\section{Conclusion}

In this article, we shown how intuitionistic logic and Kelsen's jurisprudence can be used to express Chisholm paradox faithfully. A key fact in providing a logical model to this paradox is that laws/norms are not taken as propositions. For example, in the explanation above on building the model, if we turn back to deontic expression of laws, we will have that $l_{1}$ is $O p$ and $l_{2}$ is $O(p \rightarrow q)$, but we cannot derive that $l_{3}$ is $O(q)$. $l_{3}$ is of course the meet $(\sqcap)$ between $l_{1}$ and $l_{2}$, as a meet it is strongly connection to $O\left(l_{1}\right) \wedge O\left(l_{2}\right) \leftrightarrow O\left(l_{1} \wedge l_{2}\right)$, which is a SDL valid formula. Thus, $l_{3}$ is the norm $O\left(l_{1} \wedge l_{2}\right)$, that is an obligation. However, now remembering what norms $l_{1}$ and $l_{2}$ are in this particular case, $l_{3}$ is the meeting $O(p) \wedge O(p \rightarrow q)$ that it is $O(p \wedge(p \rightarrow q))$. This conclusion, however, does not entail that in $l_{3}$ can be identified with $O(q)$, since our implication is the intuitionistic implication. This very last aspect of joining Kelsen jurisprudence and iALC also helps to avoid other deontic paradoxes.

Jorgensen's Dilemma (Jorgensen, 1937) offers a question, in fact, a dilemma, whether there is, in fact, any deontic logic. The question follows this path: 1) Norms/laws deal with evaluative sentences; 2) Evaluative sentences are not the kind of sentence that can be true or false; 3) Thus, how there is a logic of evaluative sentences? 4) Logic has as goal to define what can be drawn from whatever, and; 5) A sentence follows from a set of sentences on a basis of the relationship between the truth of the sentences in question. Thus, there is no deontic logic. What we have shown in this article, is that deontic logic is possible by considering the logic of norms as a logic on norms, instead. This reading is just what we do in legal ontologies.

We have to touch some aspects that are very well-known in the deontic approach. One is the deontic concept of permission. This case is modeled by observing that in a society regulated by law, permission is nothing more than an obligation of the State. The State promulgates what is allowed. Concerning prohibitions, the foundation is analogous. However, some subtle and theoretical problems may arise if one wants to recover the definition of forbidden $(F)$ regarding the very well-known duality $F(p) \equiv O(\neg p)$. This discussion will be the subject of another article.

On the more practical side, we have been experimenting with applications of iALC aiming to develop a methodology for law formalization. The OAB (Ordem dos Advogados do Brasil, the Order of Attorneys of Brazil) Exam can be considered an excellent choice for analyzing the performance of a system with the goal to reason in the legal world. Similarly to the US Bar Exam, it evaluates whether the candidates have the aptitude to practice the law. We have conducted three experiments in question answering on the OAB national exams (Delfino, Cuconato, Haeusler, \& Rademaker, 2017; Alkmim, Haeusler, \& Rademaker, 2018). In these articles we explain the basic structure of the OAB Exam followed by the formal justification of the corrected answers of some questions. In the future, we intend to make these results as general and algorithmic as possible, aided by a reasoner for iALC and combined with natural language processing techniques. 


\section{Bibliography}

Alchourron, C. E., \& Martino, A. (1990). Logic without truth. Ratio Juris, 3(1), 46-67. Alkmim, B., Haeusler, E. H., \& Rademaker, A. (2018). Utilizing ialc to formalize the brazilian oab exam. In Proceedings from the explainable ai in law workshop (xaila). CEUR-WS. (to appear)

Bellin, G., de Paiva, V., \& Ritter, E. (2001). Extended curry-howard correspondence for a basic constructive modal logic. In Procs of methods for the modalities.

Braüner, T., \& de Paiva, V. (2006). Intuitionistic hybrid logic. JAL, 4 (3), 231-255.

Buss, S. R. (1998). Handbook of proof theory. Elsevier.

Clément, I. (2008). Proof theoretical foundations for constructive description logic (Unpublished master's thesis). School of Computer Science. McGill University, Montrel.

Delfino, P., Cuconato, B., Haeusler, E. H., \& Rademaker, A. (2017). Passing the brazilian oab exam: Data preparation and some experiments. In A. Wyner \& G. Casini (Eds.), Legal knowledge and information systems (Vol. 302). (The 30th International Conference on Legal Knowledge and Information Systems (JURIX 2017). Expanded version at https://arxiv.org/abs/1712.05128)

de Paiva, V. (2006). Constructive description logics: what, why and how. In Context representation and reasoning.

de Paiva, V., Hausler, E. H., \& Rademaker, A. (2010). Constructive description logic: Hybrid-style. In Proceedings of hybrid logic and applications (HyLo 2010).

Fischer-Servi, G. (1984). Axiomatizations for some intuitionistic modal logics. Rendiconti del Seminario Matematico Università e Politecnico di Torino, 42.

Girard, J., Taylor, P., \& Lafont, Y. (1989). Proofs and types. Cambridge University Press.

Haeusler, E. H., de Paiva, V., \& Rademaker, A. (2010). Intuitionistic logic and legal ontologies. In Proc. jurix 2010 (pp. 155-158). IOS Press.

Haeusler, E. H., de Paiva, V., \& Rademaker, A. (2010). Using intuitionistic logic as a basis for legal ontologies. In Proceedings of the 4th workshop on legal ontologies and artificial intelligence techniques (pp. 69-76). Fiesole, Florence, Italy: European University Institute.

Haeusler, E. H., de Paiva, V., \& Rademaker, A. (2011). Intuitionistic description logic and legal reasoning. In Proceedings of international workshop data, logic and inconsistency with dexa 2011.

Haeusler, E. H., Paiva, V. D., \& Rademaker, A. (2010). Using intuitionistic logic as a basis for legal ontologies. Informatica e Diritto, XIX(1-2), 289-298.

Hughes, G. (1971). Validity and the basic norm. California Law Review, 59(3), 695-715. Jorgensen, J. (1937). Imperatives and logic. Erkenntnis, 7, 288-296.

Kelsen, H. (1946). General theory of law and state. Harvard University Press. (Translation from german by Wedberg, A. and Kraus, W.H.)

Kelsen, H. (1967). Pure theory of law [Book]. Berkeley : University of California Press. (Translated from Reine Rechtslehre, second edition, 1960) 
Kelsen, H. (1991). General theory of norms. USA: Oxford Univ. Press.

M.E. Coniglio, N. P. (2009). A paraconsistent approach to chisholm's paradox. Principia, 13(3), 299-326.

Mendler, M., \& Scheele, S. (2010). Towards constructive DL for abstraction and refinement. JAR, 44(3), 207-243. (Proc. 21st International DL Workshop)

Negri, S., \& Von Plato, J. (2008). Structural proof theory. Cambridge University Press.

Plotkin, G., \& Stirling, C. (1986). A framework for intuitionistic modal logics: extended abstract. In Proceedings of the 1986 conference on theoretical aspects of reasoning about knowledge (pp. 399-406). San Francisco, CA, USA: Morgan Kaufmann Publishers Inc. Retrieved from http://dl.acm.org/citation.cfm?id=1029786 .1029823

Rademaker, A. (2012). A proof theory for description logics. Springer.

Ranalter, K. (2010). Embedding constructive $k$ into intuitionistic $k$. Electr. Notes Theor. Comput. Sci., 262, 205-219.

Raz, J. (1972). Legal principles and the limits of law. Yale Law Journal, 81, 823-854.

Simpson, A. (1993). The proof theory and semantics of intuitionistic modal logic (Unpublished doctoral dissertation). University of Edinburgh.

Takeuti, G. (2013). Proof theory. Courier Dover Publications.

Valente, A. (1995). Legal knowledge engineering: A modelling approach. IOS Press. (Amsterdam) 